\title{
Data Queuing in GPRS/4G Networks
}

\author{
Jerry Pylarinos \\ Department of Electrical \& Computer \\ Engineering, \\ University of Patras, \\ Rion 26500, Patras, Hellas. \\ Tel: +302610997301 \\ gpyl@gmail.com
}

\author{
Spiros Louvros \\ Department of Telecommunication \\ Systems \& Networks, \\ Technical Educational Institute of \\ Messologi \\ National road Nafpaktos -Antirio, \\ Perioxi Varia, Nafpaktos \\ slouvros@teimes.gr
}

\author{
Stavros Kotsopoulos \\ Department of Electrical \& Computer \\ Engineering, \\ University of Patras, \\ Rion 26500, Patras, Hellas. \\ Tel: +302610997301 \\ kotsop@ece.upatras.gr
}

\author{
George Asimakopoulos \\ Systems \& Networks, \\ Technical Educational Institute of \\ Messologi \\ National road Nafpaktos -Antirio, \\ Perioxi Varia, Nafpaktos \\ gasimakop@gmail.com
}

\begin{abstract}
The performance of radio resource allocation in GPRS network is critical for the dimensioning and the optimization of next generation 4G networks with GPRS capacity. Previous work has analyzed this problem, however in the present work a two dimension Markov chain, using guard channels for handover calls and priority for voice calls to pre-empt GPRS data packets is assumed. Moreover a queue is considered to be valid only for the pre-empted data packets.
\end{abstract}

\section{Keywords}

GPRS, 4G, voice pre-emption.

\section{INTRODUCTION}

GPRS network is considered to be a complementary network to GSM, dedicated for data packet communications. Moreover in next generation cellular networks, like 4G, the GSM/GPRS is just an access network, figure 1 . The general aspect is that high data access, through the 4G/3G and WiFi coverage, will be available in many non overlapping hotspot areas while the whole coverage area will provide data access through GPRS. The general idea is that a cellular data customer can use the resources of WLAN whenever possible in order to increase its data rates and to make use of all the available multimedia services without delays.

Permission to make digital or hard copies of all or part of this work for personal or classroom use is granted without fee provided that copies are not made or distributed for profit or commercial advantage and that copies bear this notice and the full citation on the first page. To copy otherwise, or republish, to post on servers or to redistribute to lists, requires prior specific permission and/or a fee.

MOBIMEDIA 2007, August 27-29, Nafpaktos, Greece

Copyright $\odot 2007$ ICST 978-963-06-2670-5

DOI 10.4108/ICST.MOBIMEDIA2007.1908

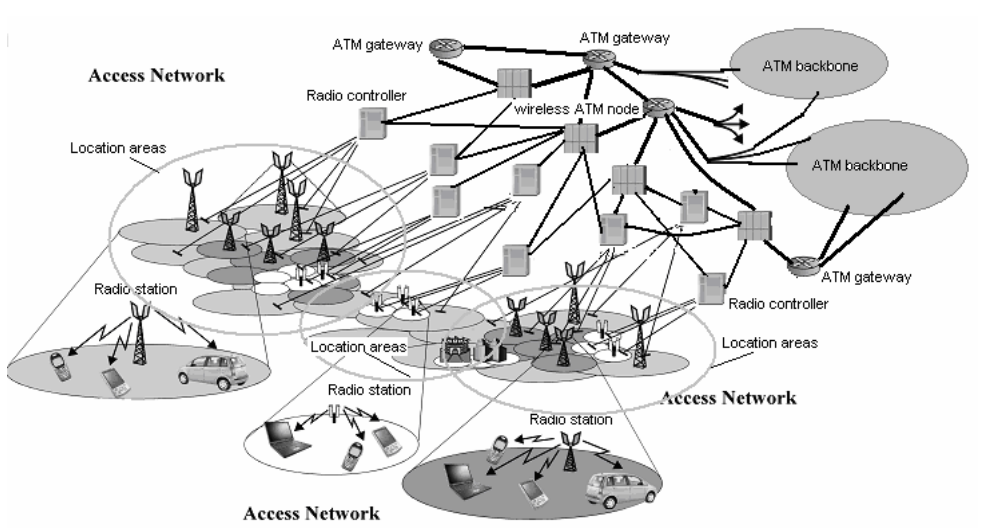

Figure 1: The wireless cellular Radio Access environment for $4 \mathrm{G}$ networks

Of course the deployment of the WLAN network is based on Hotspots architecture. Hence the coverage of WLAN network will be limited in special places, where most of the customers can use a laptop or a PDA to use the offered services. Such places are commercial centres, city centre areas like cafeterias or hotels, enterprises buildings or airports and finally crowded domestic areas. There is no need for full coverage of the whole geographical area of a city or a country, since in the areas of no WLAN coverage GPRS solution is enough to provide the mobility freedom to cellular customers with satisfactory moderate data rates. In such a case the cellular customer might start a session with the best network coverage, say WLAN in a hotspot area. While the customer is waiting, he can take advantage of the high bandwidth of WLAN in order to gain access to the offered services. As the customer moves away from the coverage area of the hotspot the end device detects the failure of service provision and seamlessly should switch the data flow to the next available data network, that is, GPRS. The important is that this flow switching is seamless to the end user, the data delay is the least 
possible by assigning special terchniques and also that when the end user gets back to a place with WLAN coverage the flow should be handed back to the WLAN network. Generally speaking, the integration of WLAN network within cellular networks depends always on the ownership or management of the WLAN network. There are two general scenarios. The first case is that the cellular operator owns, and is responsible, for the management of the WLAN network. In such a case the operator has the advantage of providing preferred billing and customer management policies. Additionally operators can use the existing cellular authentication and billing mechanisms in the customers that are using the WLAN access network. The other case is that the WLAN is not owned by the cellular operator but from a WISP. In such a case there must be certain roaming contracts in order to allow the usage of the WLAN access network in certain hotspots. In this solution is more difficult to implement the seamless session and flow switching, as discussed earlier, since in most cases of roaming the IP based session should be broken down and restarted

The integration of WLAN with cellular networks has been specified by the ETSI [1], [2] in two general approaches, the so called loose coupling and the tight coupling. In loose coupling approach the deployment of WLAN is designed as a complementary access network to GPRS, using the subscriber GPRS databases without using the core network [1]. In figure 2 the GPRS -WLAN interconnection architecture is presented.

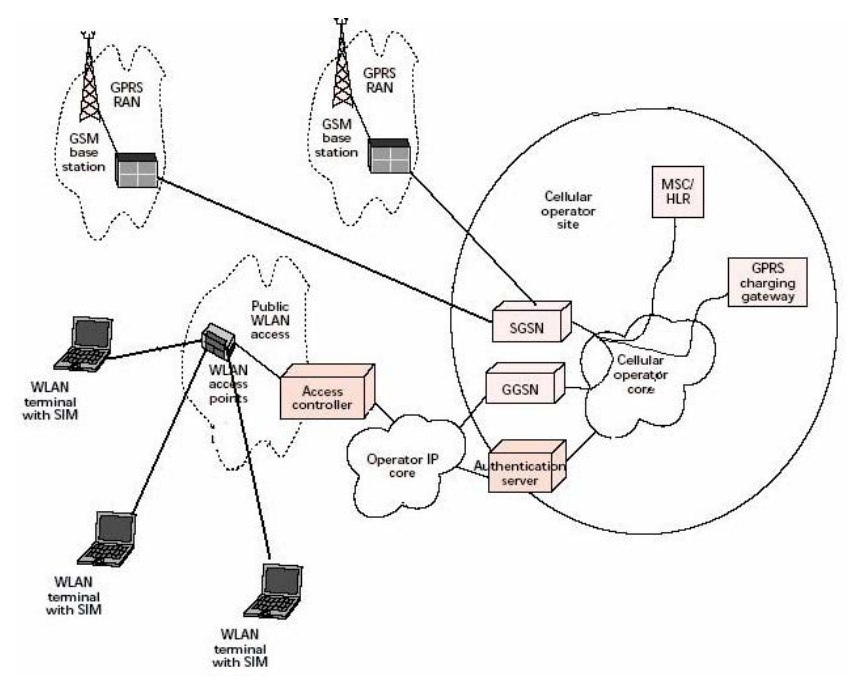

Figure 2: The GPRS - WLAN interconnection for 4G networks

The interface connecting GPRS to other IP networks bypassing the core elements (SGSN, GGSN) is the Gi interface from GGSN to PDN, which is also connected to database HLR. Hence it is obvious that the loose coupling would be to the Gi interface. Tight coupling integrates WLAN network towards the GPRS core network in the same way as any other access network. Access networks are connected to the core elements bypassing all other wireless radio environments. In Figure 2 the interface to GPRS core network element (SGSN) is the Gb interface.

The current preferred solution by the operators seems to be the loose coupling, using a USIM based authentication and billing. With this solution the customer may use his USIM card to access a set of wireless data services over WLAN network. There are also two main advantages for using the loose coupling against tight coupling. First of all the WLAN network may be owned by a WISP operator and in that case roaming / billing agreements have to be enabled through a dedicated connection between cellular operator and WLAN operator. In that case the interoperability is guaranteed and there is no need for a cellular operator to deploy also a WLAN network and to invest more for the services. On the other hand tight coupling exists only when the WLAN network is owned and managed by the cellular operator, since the integration is inside the cellular architecture. Second it is not necessary to introduce cellular technology firmware in the WLAN network as it is with the tight coupling

\section{TRAFFIC MODEL WITH DATA PACKET PRE-EMPTION}

General Packet Radio Service (GPRS) [3] is exploited over the existing GSM Radio access network. In [4] a reservation strategy is proposed only for data packet channels and the impact of the reservation number in the mixed voice - data performance is analyzed. In [5] different solution strategies for GPRS network performance are considered and certain partition strategies are proposed to divide cell capacity between GSM and GPRS traffic. Moreover in [6] guard channels prioritizes the GSM voice handoff calls but it has the drawback of low channel utilization. The GSM/GPRS network uses the same TDMA/FDMA structure and each physical channel can be assigned to either GPRS or GSM traffic. One or several physical channels can be allocated to a mobile station at a time on a demand basis. As a result the temporary allocation of these guard channels to GPRS users can improve the channel utilization. In [7] a first approach to the study of mixed traffic from GSM and GPRS sources has been presented. In this paper a parametric two one-dimensional Markov chains solution is presented, one for GSM and one for GPRS, and the results are explained for future network optimization. In the present work the radio resources are completely shared by voice calls and GPRS data packets (the complete sharing strategy [5] is adopted). Moreover the performance of radio resource allocation in GSM/GPRS, using pre-emption of GPRS packets upon request from GSM voice traffic, is evaluated without using a parametric relation but based on a two dimensional Markov chain. Finally, in order to reserve the QoS for data packets in the presence of preemption, a queue is considered to exist for the pre-empted data packets.

The analytical model is based on a two-dimensional Markov chain. The analysis will be focused on a single cell in isolation and assume that the network is symmetric and the traffic is homogenous. Let the state $(i, j)$ denote that there are $i$ voice calls and $j$ GPRS packets in the system. Pij denotes the probability that the system is in state $(i, j)$. It is also assumed that the total number of channels in a cell is $C$ and the number of guard channels reserved to prioritize voice handoff calls is $G$. The arrival of new and handoff voice call requests follow a Poisson processes with rates $\lambda_{c}$ and $\lambda_{h}$ respectively with equal arrival rate while the service time of new and handover calls is assumed to be exponentially distributed with a mean of $1 / \mu_{v}$. For GPRS data the arrival of new data packets is also assumed to be a Poisson 
process with rate $\lambda_{d}$. The GPRS data packets service time is also considered to be exponentially distributed with a mean of $1 / \mu_{d}$.

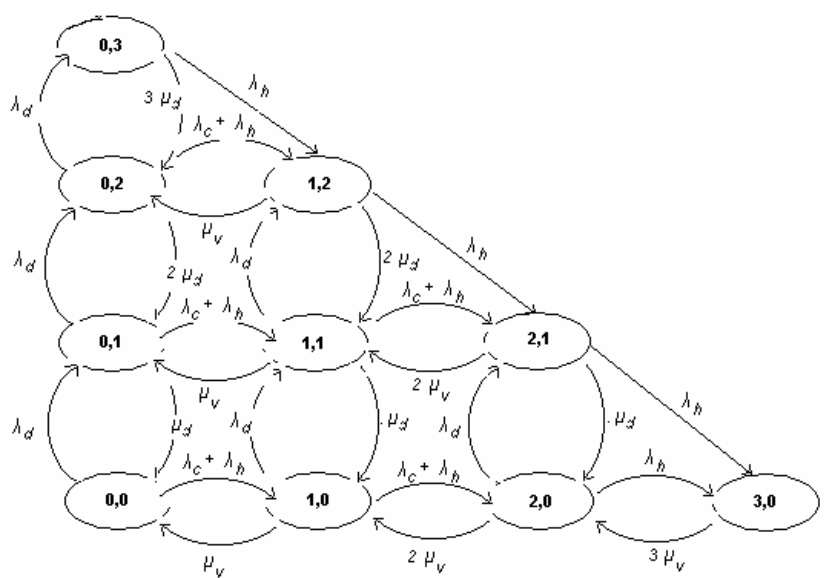

Figure 3: The state diagram (two dimensional Markov chain) for GSM/GPRS traffic

Figure 3 presents the case of a two dimensional Markov chain for $C=3$ and $G=1$. In the more general case there is no restriction in the numbers of available channels and reserved guard channels. For the more general case the idea of state feasibility has been introduced, where $\gamma_{\mathrm{ij}}$ represents a binary feasibility indicator of the chain state. When $\gamma(i, j)$ equals 1 the $(i, j)$ state is feasible otherwise it is unfeasible. According to Figure 3, GPRS packets are blocked when there are no channels available and also the pre-empted packets by voice arrivals will be dropped. The state diagram equation from Figure 3 is:

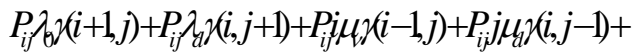

$+P_{i j} \lambda_{2}(i+1, j-1)=P_{i \rightarrow j} \lambda_{2}(i-1, j)+P_{i j-1} \lambda_{i}(i, j-1)+P_{i+j}(i+1) \mu_{2}(i+1, j)+$

$\left.\left.+P_{i j 1}(j+1) \mu_{i}\right)(i, j+1)+P_{i-j+1} \lambda_{3}\right)(i-1, j+1)$.

where,

$$
\begin{aligned}
& \lambda_{0}=\left\{\begin{array}{cc}
\lambda_{c}+\lambda_{h} & i<C-G \\
\lambda_{h} & i \geq C-G
\end{array}\right. \\
& \lambda_{1}=\left\{\begin{array}{cc}
\lambda_{c}+\lambda_{h} & i<C-G \text { AND } i+j=C \\
\lambda_{h} & C-G \leq i<C \text { AND } i+j=C \\
0 & \text { otherwise }
\end{array}\right. \\
& \lambda_{2}=\left\{\begin{array}{cc}
\lambda_{c}+\lambda_{h} & i \leq C-G \\
\lambda_{h} & i>C-G
\end{array}\right.
\end{aligned}
$$

$\lambda_{3}=\left\{\begin{array}{cc}\lambda_{c}+\lambda_{h} & i \leq C-G \text { AND } i+j=C \\ \lambda_{h} & C-G<i<C A N D i+j=C \\ 0 & \text { otherwise }\end{array}\right.$

and the condition for overall probability equals to unity:

$\sum_{\text {over all } \gamma_{i j}} P_{i j}=1$

From Figure 3 the blocking probability of GPRS data packets $P_{G b}$ and the pre-emption probability $P_{G p}$ of GPRS packets from voice calls are evaluated for the general case as:

$P_{G b}=\sum_{i+j=C} P_{i j}$

$P_{G p}=\frac{\lambda_{1}}{\lambda_{d}\left(1-P_{G b}\right)}\left(\sum_{i+j=C} P_{i j}\right)$

\section{PROPOSED MODEL WITH PACKET QUEUE FOR PRE-EMTED TRAFFIC}

In this case it is considered to implement a queue $\mathrm{Q}$ for the rejected packets from voice pre-emption. Figure 4 shows again the case of a two dimensional Markov chain for $C=3, Q=2$ and $G=$ 1. According to Figure 4, new GPRS packets are blocked when there are no channels available while the pre-empted packets, by voice arrivals, will be dropped only when there is no available channel in the queue.

The state diagram equation from Figure 4 is:

$P_{i j} \lambda_{2}(i+1, j)+P_{i j} \lambda_{1}(i, j+1)+P_{i j} \mu_{2},(i-1, j)+P_{i j} j \mu_{l} \gamma(i, j-1)+$

$\left.+P_{i j} \lambda_{i}(i+1, j-1)=P_{i-1 j} \lambda_{2}(i-1, j)+P_{i j-1} \lambda_{3}(i, j-1)+P_{i+j}(i+1) \mu_{2}\right)(i+1, j)+$

$+P_{i j+1}(j+1) \mu_{i}(i, j+1)+P_{i-j j+1} \lambda_{i}(i-1, j+1)$.

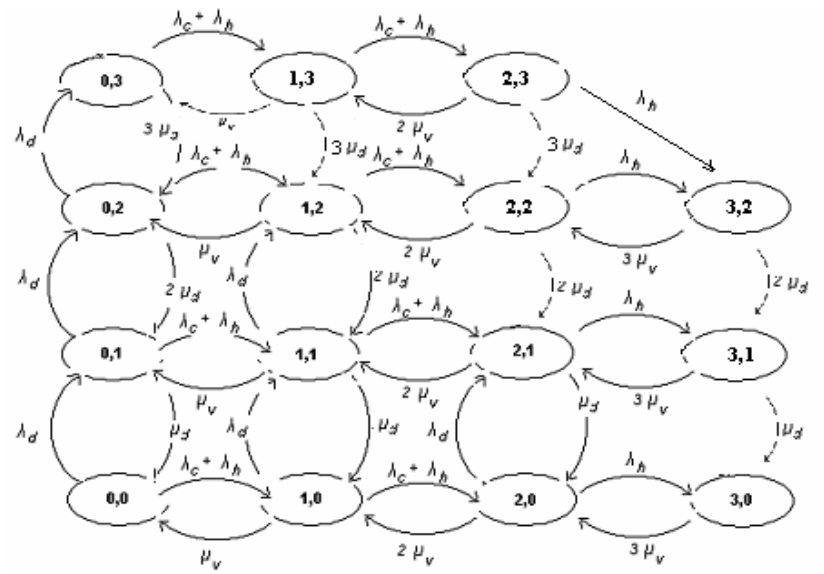


Figure 4: GSM/GPRS model with pre-empted packet queue for $\mathrm{C}=3, \mathrm{G}=1, \mathrm{Q}=2$

where,

$\lambda_{0}=\left\{\begin{array}{cc}\lambda_{c}+\lambda_{h} & i<C-G \\ \lambda_{h} & i \geq C-G\end{array}\right.$

$\lambda_{1}=\left\{\begin{array}{cc}\lambda_{d} & j \leq B \text { AND } i+j<C \\ 0 & \text { otherwise }\end{array}\right.$

$\lambda_{2}=\left\{\begin{array}{cc}\lambda_{c}+\lambda_{h} & i \leq C-G \\ \lambda_{h} & i>C-G\end{array}\right.$

$\lambda_{3}=\left\{\begin{array}{cc}\lambda_{d} & j<B \text { AND } i+j<C \\ 0 & \text { otherwise }\end{array}\right.$

and the constrain condition:

$\sum_{\text {over all } \gamma_{i j}} P_{i j}=1$

The blocking probability of GPRS data packets $P_{G b}$ and the preemption probability $P_{G p}$ of GPRS packets from voice calls are evaluated, considering the general case of $C$ channels and $G$ guard channels, as:

$$
\begin{aligned}
& P_{G b}=\sum_{i+j \geq C} P_{i j} \\
& P_{G p}=\frac{1}{\lambda_{d}\left(1-P_{G b}\right)}\left[\sum_{\substack{i+j \geq C \\
\text { and } \\
i<C-G}}\left(\lambda_{c}+\lambda_{h}\right) P_{i j}+\sum_{\substack{i+j \geq C \\
\text { and } \\
C-G \leq i<C}} \lambda_{h} P_{i j}\right]
\end{aligned}
$$

The mean queue delay time of GPRS data packets is

$$
D=\frac{\sum_{i=0}^{C} \sum_{J=1}^{C} j P_{i j}}{\lambda_{d}\left(1-P_{G b}\right)}-\frac{1}{\mu_{d}}
$$

\section{NUMERICAL RESULTS}

A typical cell configuration in a city centre was considered with 28 channels. In that case the number of guard channels $G$ is considered to be 1 . Since we are interested in the GPRS performance, typical values (from operator's network in Greece) for mean new and handoff arrival rate is taken to be 0.07 calls $/ \mathrm{sec}$ and 0.05 respectively, and the mean service time of new or handover calls is considered to be 180 seconds. Arrival rate of GPRS data packets is a system parameter and a fixed value for the mean service time $\mu_{d}$ of GPRS data packets is considered to be 2 seconds, while the queue size $\mathrm{Q}=28$.

Figure 5 presents the GPRS pre-emption probability vs. GPRS traffic. In that case the dependence is linear indicating a smooth increment on GPRS traffic intensity, a typical case in a city centre.

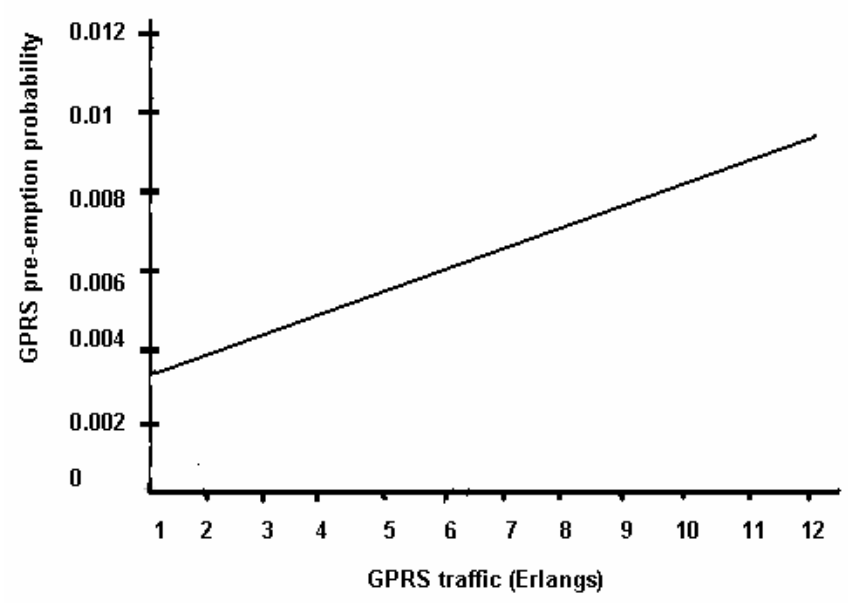

Figure 5: GPRS pre-emption probability vs. GPRS traffic for the case without packet queue

Figure 6 presents the GPRS pre-emption probability vs. GPRS traffic in case there is a queue for the pre-empted packets. In that case the dependence is again linear, indicating a smooth increment on GPRS traffic intensity. However it has better performance than the case without packet queue.

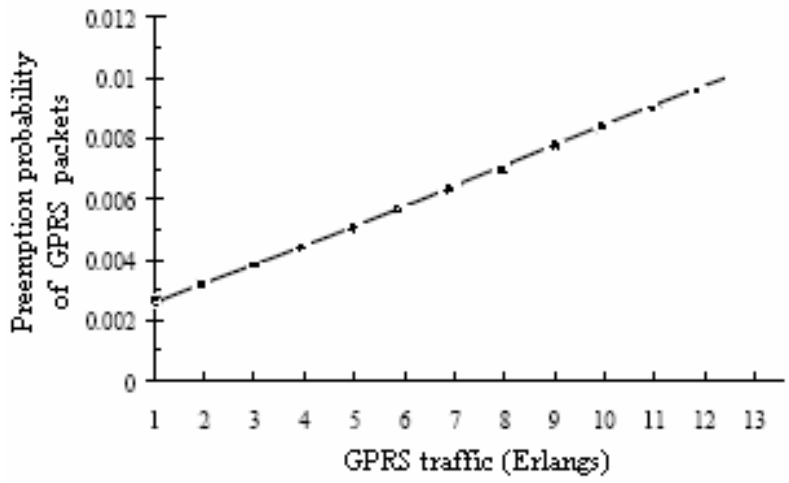


Figure 6: GPRS pre-emption probability vs. GPRS traffic with queued GPRS packet

Figure 7 presents the mean queue delay of pre-empted GPRS packets from equation (9). It is interesting to see the non linear dependence on GPRS traffic intensity. For low GPRS traffic the mean waiting time is high since when a packet is pre-empted it has to wait for a voice call to complete and the mean voice call service time is higher than data. As the GPRS traffic load is increased more channels are reserved for GPRS traffic. Hence for preempted packets they have to wait either for a voice call to complete or for a data packet to be served. Since data packets are served faster than voice (the mean service time of data packets is much smaller than voice) the pre-empted packets will be served faster and mean waiting time in queue is lower.

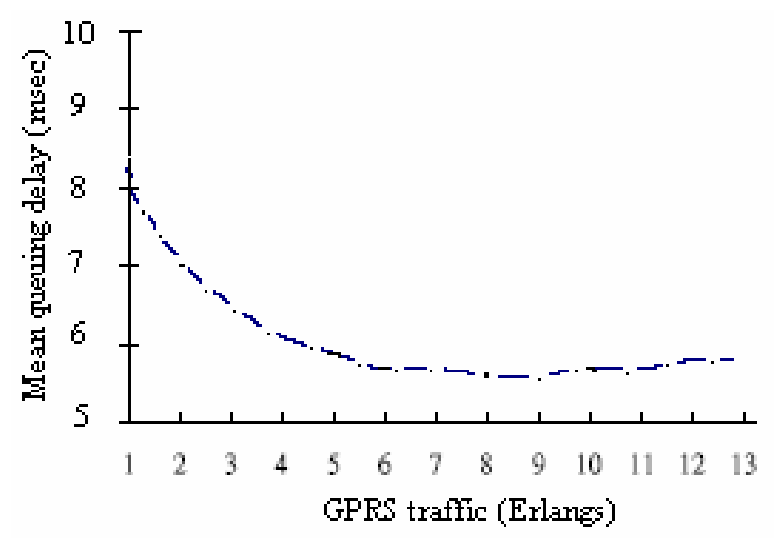

Figure 7: Mean queue delay for GPRS packets

\section{REFERENCES}

[1] ETSI, "requirements and Architectures for Interworking between HIPERLAN/3 and $3^{\text {rd }}$ Generation cellular Systems" Technical Report ETSI TR 101957

[2] 3GPP "Feasibility Study on 3GPP System to WLAN Interworking”, Technical Report eGPP TR 22.934 v1.2.0

[3] ETSI, “GSM 03.60 General packet radio service (GPRS) : Service description, Stage 2,” v. 5.2.0, Jan. 1998.

[4] C. Lindemann and A. Thummler, "Performance analysis of the general packet radio service," 21st International Conference on Distributed Computing Systems, pp. 673-680, Mesa, AZ, USA, April 2001

[5] M. Ermel, K. Begain, T. Muller, J. Schuler, and M. Schweigel, "Analytical comparison of different GPRS introduction strategies," Proc. 3rd ACM Int. Workshop on Modeling Analysis and Simulation of Wireless and Mobile Systems, pp. 3-10, Boston, USA, Aug. 2000.

[6] D. Hong and S. S. Rappaport, "Traffic model and performance analysis for cellular mobile radio telephone systems with prioritized and no-protection handoff procedure," IEEE Trans. Veh. Technol., vol. 35, pp. 77-91, Aug. 1985

[7] S. Ni and S. G. Haggman, "GPRS performance estimation in GSM circuit switched services and GPRS shared resource systems," Proceedings of IEEE WCNC'99, vol. 3, pp. 1417-1421, New Orleans, USA, Sep. 1999. 